\title{
The selective electrochemical sensing of dopamine at a polypyrrole film doped with an anionic $\beta$-cyclodextrin
}

\author{
Claire C. Harley, Valeria Annibaldi, Tian Yu, Carmel B. Breslin \\ Department of Chemistry, National University of Ireland Maynooth, Maynooth, Co. Kildare, Ireland
}

\section{A R T I C L E I N F O}

\section{Keywords:}

Dopamine sensor

Sulfonated $\beta$-cyclodextrin

Ascorbic acid

Uric acid

Selectivity

Sensitivity

\begin{abstract}
A B S T R A C T
Pyrrole was electropolymerised in a $0.01 \mathrm{M}$ sulfonated $\beta$-cyclodextrin solution to generate an adherent polypyrrole film doped with the anionic sulfonated $\beta$-cyclodextrin, PPy-S $\beta C D$. This polymeric material was used as an electrochemical sensor for the detection of dopamine (DA), and compared with the sensing abilities of polypyrrole doped with several more common anionic dopants. The sensing performance of the PPy-S $\beta C D$ film was significantly better, with a linear calibration curve extending to $50 \mu \mathrm{M}$, with a sensitivity of $0.90 \mu \mathrm{A} \mu \mathrm{M}^{-1} \mathrm{~cm}^{-2}$ and a detection limit of $1.0 \times 10^{-6} \mathrm{M}$. Excellent selectivity was achieved and no interference was observed from a range of interference compounds, including ascorbic acid, uric acid, aspartic acid, acetylcholine, aminobutyric acid, glutamic acid, glycine, histamine, acetaminophenol 5-hydroxytryptamine and 5-hydroxyindole acetic acid. However, interference was seen with the structurally-related epinephrine (Ep) and 3,4-dihydroxyphenylacetic acid (DOPAC), with the oxidation of DA, Ep and DOPAC occurring at similar potentials. The good selectivity in the presence of the other interference species was attributed to an interaction between the cyclodextrin dopant and the protonated DA molecule which was evident when data were fitted to Michaelis-Menten and Lineweaver-Burk kinetics.
\end{abstract}

\section{Introduction}

Dopamine (DA) is an important molecule in the catecholamine family and plays a significant role in the central nervous, cardiovascular and renal systems [1]. It is not surprising that the quantification of DA in biological fluids is the topic of much attention. As DA is easily oxidised to generate the dopamine_o_quinone, there is much interest in the development of simple and efficient electrochemical sensors for the analysis of DA levels. However, the electrochemical detection of DA is complicated by the co-existence of a number of other redox active species, such as ascorbic acid (AA) and uric acid (UA), which oxidise at similar potentials to that of DA at most electrodes $[2,3]$.

These issues have been partially resolved by using modified electrodes and Nafion, a permselective membrane, has been commonly used to repel the negatively charged ascorbate anion from the electrode surface, enabling the detection of dopamine without significant interference from the oxidation of ascorbate. In many cases, a Nafion layer is deposited onto an electroactive material that is capable of oxidising DA, giving rise to an outer layer with good selectivity properties [4-7]. This approach is based on the ionic forms of DA and AA at physiological $\mathrm{pH}$. The AA exists in the anionic form, with a pKa of 4.10, while DA is in the cationic form, with a $\mathrm{p} K_{\mathrm{a}}$ of 8.87 .

Alternatively, negatively charged layers can be formed, which also serve to repel the anionic AA. For example, Zheng and Zhou [8] have used dodecyl sulfate-modified carbon paste electrodes to selectively sense DA in the presence of AA. Again, the selectivity was explained in terms of a high density of negatively charged groups at the interface. More recently, the electrochemical detection of DA was obtained using positively charged sheets of $\mathrm{Zn}-\mathrm{NiAl}$ layered double hydroxide with negatively charged layers of reduced graphene oxide [9].

Graphene-based materials have also been used [10-12]. For example, Shang et al. [11] have shown that multilayer graphene nanoflake films can be used to discriminate between AA, DA and UA, with a detection limit of $0.17 \mathrm{mM}$ for $\mathrm{DA}$, while graphene modified overoxidised polypyrrole electrodes have been prepared and applied in the electrochemical detection of dopamine [13]. Immobilised nanoparticles have also been reported in the sensing of dopamine, for example, Mathiyarasu et al. [14] used gold nanoparticles deposited at PEDOT to selectively discriminate between AA, DA and UA. The AA, DA and UA oxidation waves were observed at approximately $-0.05 \mathrm{~V}, 0.16 \mathrm{~V}$ and $0.30 \mathrm{~V}(\mathrm{Ag} \mid \mathrm{AgCl})$, respectively. Gold nanoparticles have also been combined with polypyrrole and reduced graphene oxide for the ultrasensitive

\footnotetext{
* Corresponding author.

E-mail address: Carmel.Breslin@mu.ie (C.B. Breslin).
} 
detection of dopamine [15].

Various polymeric films, such as polyaniline and polypyrrole, have been used in the selective detection of DA in an excess of AA. In particular, polypyrrole shows permselective properties in the over-oxidised state, as groups, such as $>\mathrm{CO}$ and $-\mathrm{COO}^{-}$, are generated providing discrimination against the anionic AA [16,17]. Electropolymerised films of 4-allyl-2-methoxyphenol (eugenol) have also been employed to determine DA in an excess of AA [18]. The high selectivity and sensitivity observed were explained in terms of charge discrimination, analyte accumulation and a catalytic mediation of redox sites. In more recent years, more complex polymeric composites have been employed, including graphene decorated with zinc oxide-copper heterostructures and polypyrrole nanofibers [19], graphene and polypyrrole 3D composites [20], poly(ionic liquids) combined with polypyrrole and graphene oxide nanosheets [21] and conducting polypyrrole hydrogels [22].

In this study, pyrrole was electropolymerised in the presence of sulfonated $\beta$-cyclodextrins, which served as the dopants, to give a simple polypyrrole film coated electrode. This electrode exhibited good sensitivity and selectivity, facilitating the oxidation of dopamine, but inhibiting the oxidation of AA, UA and a wide range of other interference species. There are various publications describing the electrochemical synthesis of polypyrrole doped with sulfonated $\beta$-cyclodextrins. Bidan and co-workers [23] and Temsamani et al. [24] have elctrosynthesised polypyrrole doped with sulfonated $\beta$-cyclodextrins, while Tamer et al. [25] have prepared a three-dimensional network of branched fibres of polypyrrole doped with sulfonated $\beta$-cyclodextrin through an interfacial polymerisation process. These anionic cyclodextrin doped polypyrrole films are particularly easy to prepare and they have been used to detect and uptake viologens [26] and in the controlled release of dopamine [27]. Also, polypyrrole has good biocompatibility properties [28] while cyclodextrins are well known in the drug delivery field [29], making these modified electrodes considerably more suitable for implantation compared to some of the more complex electrodes. We have previously shown that it is possible to detect DA at polypyrrole doped with a sulfonated $\beta$-cyclodextrin [30]. A more detailed study, where the composition of the solution phase, ionic strength, buffering action, and the presence of a wide range of interference molecules together with the mode of DA sensing, is now considered and discussed.

\section{Experimental}

All chemicals were obtained from Aldrich and were of analytical grade reagents. The pyrrole monomer was distilled before use and stored at $-4{ }^{\circ} \mathrm{C}$. The $s \beta-\mathrm{CD}$ was purified using a Schlenk line by dissolving the sample in a small volume of deionised water and then the sample was dried at $70^{\circ} \mathrm{C}$ under vacuum. All other chemicals were used without any further purification.

The electrochemical data, including cyclic voltammetry, constant potential amperometry and rotating disc voltammetry, were recorded with a Solartron 1287 potentiostat. Platinum $(99.99 \%, \mathrm{~d}=3.0 \mathrm{~mm})$ disc electrodes were used and these were combined with a high surface area platinum wire, which served as the counter electrode, and a saturated calomel electrode (SCE), as the reference, in a three electrode cell. An EG\&G, Model 636 ring disc glassy carbon (GC) electrode was used to record the rotating disc voltammetry. The disc electrodes were prepared by embedding the metal rod in epoxy resin which was supported in a Teflon holder and electrical contact was achieved by threading a wire to the other end of the rod. The exposed surfaces were polished using successively smaller sizes of diamond paste to give a mirror-like finish. The electrodes were then cleaned in an ultrasonic bath to remove any residues from polishing. The cyclic voltammograms were recorded at room temperature in deoxygenated solutions at a scan rate of $100 \mathrm{mV} \mathrm{s}^{-1}$. A $0.1 \mathrm{M} \mathrm{Na}_{2} \mathrm{SO}_{4}$ or $0.1 \mathrm{M} \mathrm{NaCl}$ supporting electrolyte with a $\mathrm{pH}$ of 7.0 was used. All solutions were deoxygenated with nitrogen.

Electrochemical quartz crystal microbalance (EQCM) measurements were performed with a $\mathrm{CH}$ instruments system, Chi440 EQCM. The polymer was formed at a gold quartz crystal electrode supplied by Cambria Scientific $\left(0.203 \mathrm{~cm}^{2}\right)$ with a platinum wire counter electrode and a custom-made $\mathrm{Ag} \mid \mathrm{AgCl}$ reference electrode. The oscillation frequency was converted to mass using the well-known Sauerbrey equation [31], Eq. (1). Here, $\Delta f$ represents the observed frequency change, $\Delta m$ corresponds to the mass change and $C_{f}$ is the sensitivity of the crystal, which was found experimentally to be $7.9897 \times 10^{8} \mathrm{~Hz} \mathrm{~cm}^{2} \mathrm{~g}^{-1}$. Thin polymer films deposited to a final charge density of $1.2 \times 10^{-2} \mathrm{C} \mathrm{cm}^{-2}$ were employed to minimise viscoelastic effects.

$\Delta m=-\frac{A \sqrt{\left(\rho_{q} \mu_{q}\right)}}{2 f_{0}^{2}} \Delta f=-\frac{1}{C_{f}} \Delta f$

A $0.20 \mathrm{M}$ pyrrole solution was combined with $0.01 \mathrm{M}$ sulfonated $\beta$-cyclodextrin and the sulfonated cyclodextrin doped polypyrrole $(\mathrm{PPy}-\mathrm{S} \beta \mathrm{CD})$ was formed at $0.80 \mathrm{~V}$ vs SCE until a fixed charge of $0.32 \mathrm{C} \mathrm{cm}^{-2}$ was passed, unless otherwise stated. No supporting electrolyte was necessary, as the anionic cyclodextrin has a sufficiently high conductivity ( $4.5 \mathrm{mS} \mathrm{cm}^{-1}$ for a $0.01 \mathrm{M}$ solution). This ensured that the polymer was only doped by the anionic cyclodextrin. For comparative purposes, polypyrrole films doped with chloride (PPy-Cl), sulfate $\left(\mathrm{PPy}-\mathrm{SO}_{4}\right)$ and dodecyl sulfate (PPy-S $\left.\beta \mathrm{DS}\right)$ were synthesised at identical applied potentials and to the same final charge, but with the appropriate salts in solution. Once the modified electrodes were formed, they were cycled in the background electrolyte, between $-0.10 \mathrm{~V}$ and $0.90 \mathrm{~V}$ vs SCE at $100 \mathrm{mV} \mathrm{s}^{-1}$ for 10 cycles to ensure the release of any pyrrole or oligomers bound within the polymer. Then, the modified electrodes were rinsed with distilled water and transferred to the DA-containing solutions.

The polymer thickness was measured using a Tencor profilometer (Tencor Veeco Dektac 6 M Stylus Profilometer), while a Hitachi scanning electron microscope was employed to study the morphology of the polymer films. An Emitech K550x gold sputter coater was used to deposit a thin gold layer prior to surface analysis.

\section{Results and discussion}

\subsection{Formation of $P P y-S \beta C D$}

The formation of PPy-S $\beta C D$ from a solution containing $0.2 \mathrm{M}$ pyrrole and $0.01 \mathrm{M} \mathrm{S} \beta \mathrm{CD}$ at $0.50 \mathrm{~V}$ vs SCE at platinum is shown in Fig. 1, where the logarithm of the current is presented as a function of time, while a typical charge-time plot recorded at a higher potential of $0.80 \mathrm{~V}$ vs SCE is shown in the inset. A near steady-state current is observed within $30 \mathrm{~s}$, indicating efficient polymerisation at the relatively low potential of $0.50 \mathrm{~V}$ in the presence of the anionic cyclodextrins without the presence of any other salts $[26,27]$. The charge-time plot recorded at $0.80 \mathrm{~V}$ vs SCE shows a near linear increase in charge with increasing polarisation times, which is again consistent with efficient polymerisation and the deposition of conducting polymer layers. The surface morphology of $\mathrm{PPy}-\mathrm{S} \beta C D$ formed at $0.80 \mathrm{~V}$ vs SCE is shown in Fig. 1(b). Although the typical globular structure is seen, a more structured and organised film, that appears to have ridges and valleys, giving a ladder-like arrangement is formed. This is consistent with the organised ladder doping mechanism proposed by Ingram et al. [32] for polypyrrole doped with aromatic polysulfonates, where channels were created within the polymer matrix and also with a report by Temsamani et al. [24]. In this case, polypyrrole was formed in the presence of perchlorate and S $\beta C D$ and it was concluded that the incorporated cyclodextrins provided a more organised system.

The thickness of the PPy-S $\beta C D$ films formed at $0.80 \mathrm{~V}$ to a final charge density of $0.32 \mathrm{C} \mathrm{cm}^{-2}$ was estimated as $690 \pm 50 \mathrm{~nm}$ using a Tencor profilometer. The film thickness was also estimated using Faraday's law, Eq. (2), where $M$ corresponds to the molecular weight of pyrrole, $A$ represents the surface area, $q$ is the charge, $z$ is the number of electrons transferred, while $\rho$, which represents the density, is taken as 
(a)

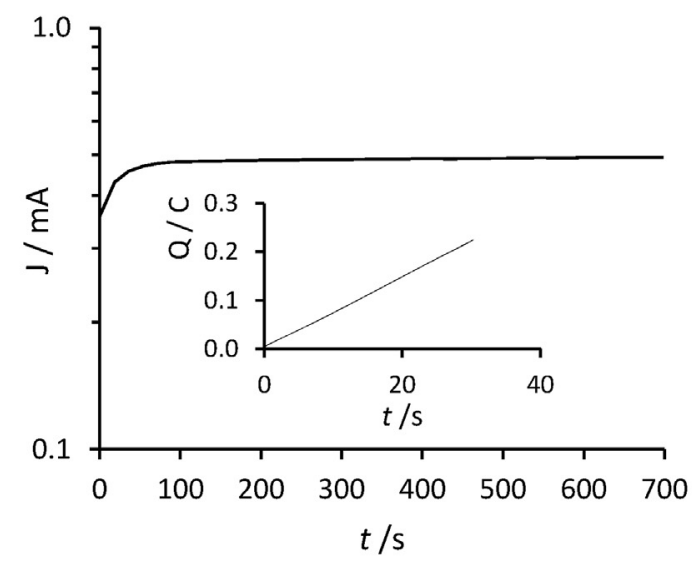

(b)

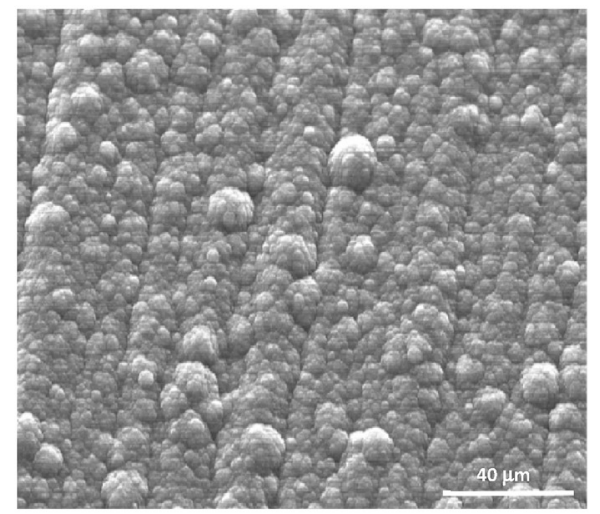

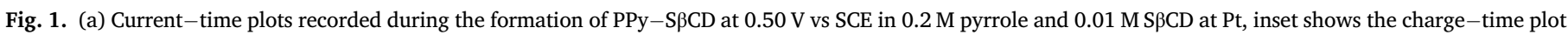
recorded at $0.80 \mathrm{~V}$ vs SCE and (b) SEM micrograph of PPy-S $\beta C D$ deposited to $1.5 \mathrm{C} \mathrm{cm}^{-2}$.

$1.5 \mathrm{~g} \mathrm{~cm}^{-3}$. In this case, the film thickness was estimated as $0.68 \mu \mathrm{m}$, which agrees very well with the experimental measurements. This is surprising given the larger size of the $S \beta C D$ dopant where in many cases, the simple expression shown in Eq. (2) fails to give a good estimate of the film thickness with large dopants [33].

$x=\frac{q M}{\rho A z F}$

\subsection{Detection of dopamine at $P P y-S \beta C D$}

Prior to the detection of DA, the PPy-S $\beta C D$ films were cycled in the background $0.1 \mathrm{M} \mathrm{Na}_{2} \mathrm{SO}_{4}$ or $0.1 \mathrm{M} \mathrm{NaCl}$ electrolytes. Once the polymer is synthesised it is likely that the $\mathrm{CD}$ cavities contain some oligomers, however when the polymer is cycled to $0.90 \mathrm{~V}$ vs SCE in the solution, the oligomers will be oxidised and released. In Fig. 2 cyclic voltammograms, recorded at $100 \mathrm{mV} \mathrm{s}^{-1}$, are shown for $\mathrm{PPy}-\mathrm{S} \beta \mathrm{CD}$ cycled in $0.1 \mathrm{M}$ $\mathrm{Na}_{2} \mathrm{SO}_{4}$ in the presence of $0.2 \mathrm{mM}$ DA and compared with polypyrrole doped with chloride, $\mathrm{PPy}-\mathrm{Cl}$, sulfate, $\mathrm{PPy}-\mathrm{SO}_{4}$, and formed in the presence of chloride anions with a neutral $\beta C D$, Fig. 2(a). The larger dodecyl sulfate doped polymer, PPy-SDS, is compared with PPy-S $\beta C D$ in Fig. 2(b). In each case the polymers were formed at $0.80 \mathrm{~V}$ vs SCE and to the same charge density of $0.32 \mathrm{C} \mathrm{cm}^{-2}$. The oxidation of DA is clearly evident with a peak oxidation potential at $0.46 \mathrm{~V}$ vs SCE, while the corresponding reduction of the dopamine-o-quinone is observed with a

(a)

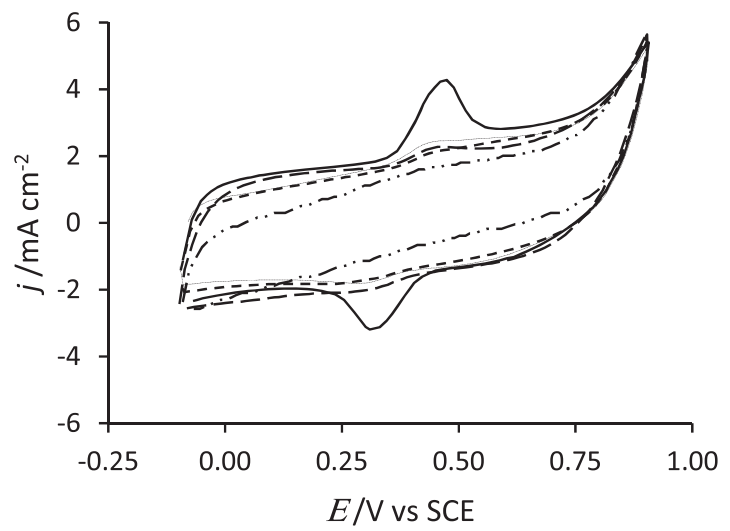

peak potential at $0.27 \mathrm{~V}$ vs SCE. A clear difference in the ability of the five modified electrodes to oxidise DA is seen. There is a significant increase in the peak currents for the oxidation of DA for the PPy-S $\beta C D$ system, compared to the other modified electrodes. Furthermore, the redox reaction at platinum (data not shown) gives a $\Delta E_{\mathrm{p}}$ value of $480 \mathrm{mV}$, while the peak separation of $190 \mathrm{mV}$, typical of quasi-reversible behaviour, is seen with the PPy-S $\beta C D$. This shows that the DA signal is from the modified polymer and not the substrate material and that the sulfonated $\beta$-cyclodextrin plays a role in the sensing of dopamine.

In order to optimise the DA oxidation peak currents, the PPy-S $\beta C D$ films were formed with different pyrrole and $S \beta C D$ concentrations, and at different applied potentials and deposited to various final charges. While the pyrrole and $S \beta C D$ concentrations had little influence, the applied potential and charge had a significant effect on the detection of DA. The influence of the applied potential employed in the formation of the polymer is illustrated in Fig. 3(a) and the influence of the charge, which gives an estimate of the film thickness, is summarised in Fig. 3(b). It is clear that the optimum current for the oxidation of DA is seen for formation potentials between $0.60 \mathrm{~V}$ and $0.83 \mathrm{~V}$ vs SCE. The lower peak currents observed when the polymer is formed at higher potentials are associated with the over-oxidation of the polymer and the formation of a more insulating polymer film [34]. At the lower applied potentials, between $0.40 \mathrm{~V}$ and $0.50 \mathrm{~V}$ vs SCE, the doping levels are lower and as the oxidation of DA is connected to the presence of the $S \beta C D$, the lower (b)

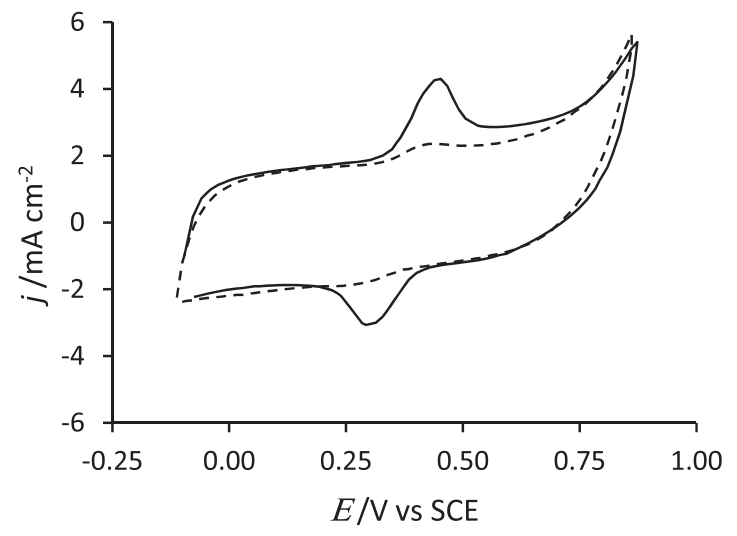

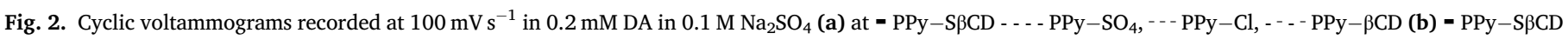
and - - PPy-SDS. 
(a)

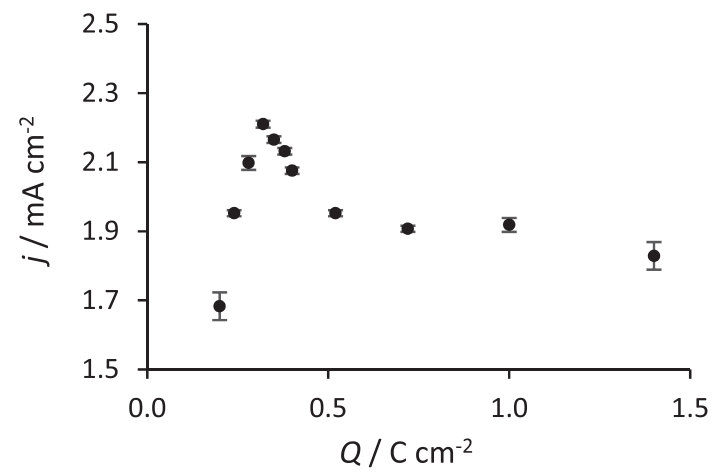

(b)

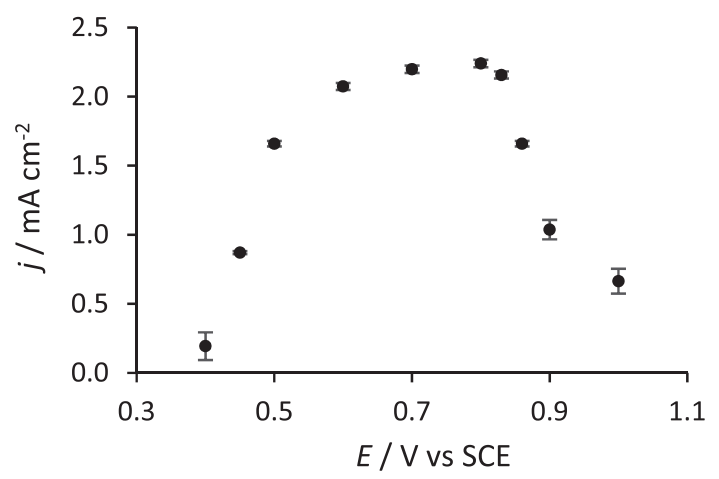

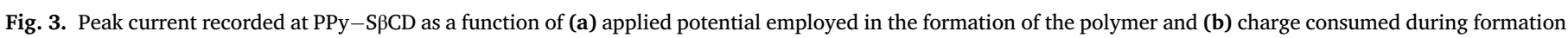
of the polymer and cycled in $0.2 \mathrm{mM}$ DA and $0.1 \mathrm{M} \mathrm{Na}_{2} \mathrm{SO}_{4}$.

doping levels result in poor detection. Likewise, higher peak currents are seen on initially increasing the charge and thickness of the polymer and again this may be related to higher concentrations of the S $\beta C D$ within the porous polymer film. However, with increasing charge, these higher oxidation currents are no longer observed and again this may be connected to the formation of more insulating polymer films as the time required for electropolymerisation increases. Indeed, similar over-oxidation effects were observed when the potential was cycled to higher potentials in the voltammetry experiments and when the polymer was polarised at $1.0 \mathrm{~V}$ vs SCE prior to the voltammetry measurements. A clear decrease in the peak current was observed when the upper potential limit was cycled to $1.0 \mathrm{~V}$ vs SCE, while a considerable change in the reversibility of the reaction, with the peak potential separation, $\Delta E_{\mathrm{p}}$, reaching $300 \mathrm{mV}$, was observed following a $10-$ min polarisation period at $1.0 \mathrm{~V}$ vs SCE. Although overoxidised polypyrrole may have good selectivity properties $[16,17]$, over-oxidation of the PPy-S $\beta C D$ film gives rise to a more insulating polymer film.

The influence of the reduction of the PPy-S $\beta C D$ film is illustrated in Fig. 4 (a), where the voltammograms are compared for PPy-S $\beta C D$ films without any reduction and polarised at $-0.25 \mathrm{~V},-0.50 \mathrm{~V}$ and $-1.00 \mathrm{~V}$ vs SCE for 10 min prior to analysis. It is clear that a prior reduction period gives rise to lower peak currents. This behaviour can be explained in terms of the cation-exchange properties of the PPy-S $\beta C D$ films $[26,34]$, where the ingress of $\mathrm{Na}^{+}$ions occurs at lower applied potentials. This is illustrated in Fig. 4 (b), where the recorded mass change is shown as a function of the applied potential as the PPy-S $\beta C D$ is cycled from $0.60 \mathrm{~V}$ to $-0.80 \mathrm{~V}$ vs $\mathrm{Ag} \mid \mathrm{AgCl}$. Reduction of the polymer begins at about $-0.20 \mathrm{~V}$ vs $\mathrm{Ag} \mid \mathrm{AgCl}$ and then the mass continues to increase as the potential is lowered to give an overall mass increase of approximately $2.5 \mu \mathrm{g}$ at $-0.80 \mathrm{~V}$ vs $\mathrm{Ag} \mid \mathrm{AgCl}$. Although some of these cations are removed on oxidation of the polymer, there is evidence to show that not all the incorporated cations are released on oxidation, giving rise to the build-up of higher salt concentrations within the polymer [35]. These events may reduce the interactions between the DA molecule and the $S \beta C D$ dopant. This observation is also consistent with variations in the structure of polypyrrole as it is cycled to low potentials and reduced. For example, Ingram et al. [32] have described polypyrrole doped with sulfonated naphthalene as a ladder-doped model where the rungs of the ladder are held in place by the positively charged polypyrrole backbone. This will provide an open porous surface, facilitating interactions between the DA molecules and the cyclodextrin dopants. However, on reduction, the structure collapses and now access to the cyclodextrin dopants is limited and this is consistent with the lower peak currents observed in Fig. 4 (a).

The influence of the nature of the supporting electrolyte is summar- (a)

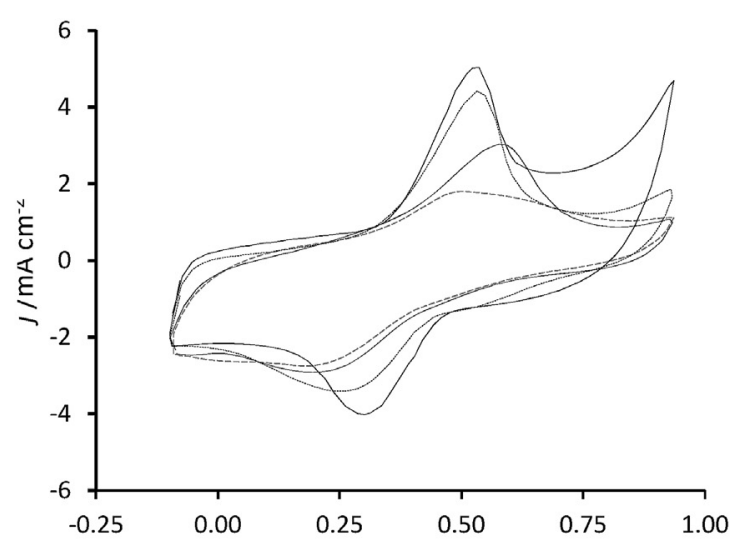

(b)

$E / \mathrm{V}$ vs SCE

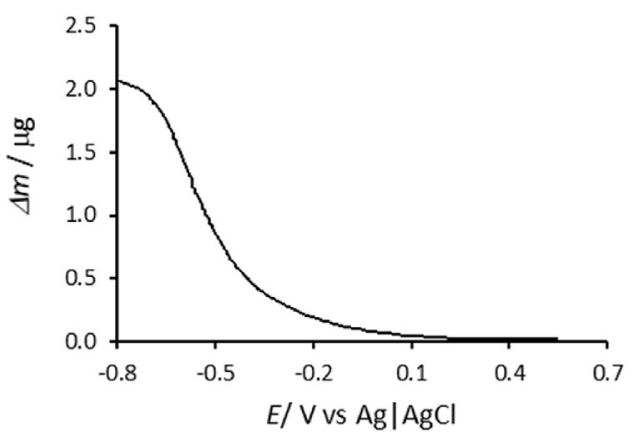

Fig. 4. (a) Cyclic voltammograms recorded at $100 \mathrm{mV} \mathrm{s}^{-1}$ in $1.0 \mathrm{mM}$ DA in $0.1 \mathrm{M} \mathrm{Na}_{2} \mathrm{SO}_{4}$ at $-\mathrm{PPy}-\mathrm{S} \beta \mathrm{CD}$ and at $\mathrm{PPy}-\mathrm{S} \beta \mathrm{CD}$ reduced at - - $-0.25 \mathrm{~V}$, $-0.50 \mathrm{~V}$ and - - - $1.0 \mathrm{~V}$ vs SCE, (b) EQCM mass-potential plot of PPy-S $\beta C D$ cycled in $0.1 \mathrm{M} \mathrm{NaCl}$ at $50 \mathrm{mV} \mathrm{s}^{-1}$.

ised in Fig. 5. The ionic strength has a clear influence on the peak potential and this is shown in Fig. 5(a) where the peak potential is plotted as a function of the ionic strength for sulfate and phosphate buffer solutions, at a fixed $\mathrm{pH}$ of 7.0. While increasing ionic strength gives rise to lower 
(a)

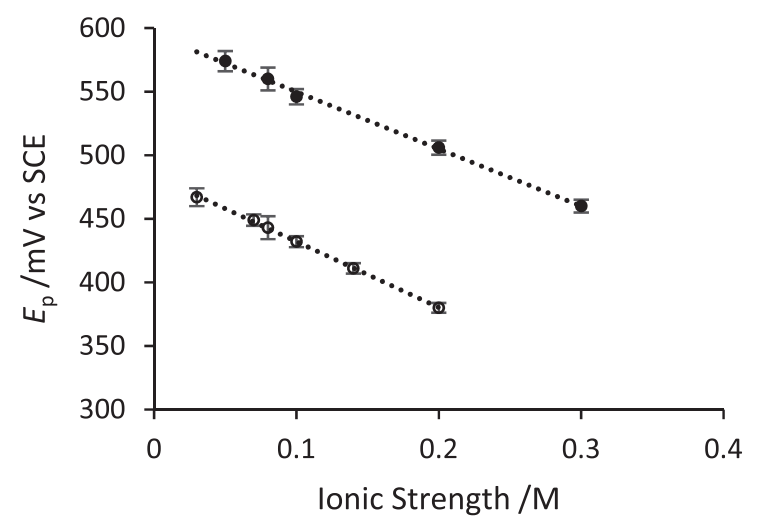

(b)

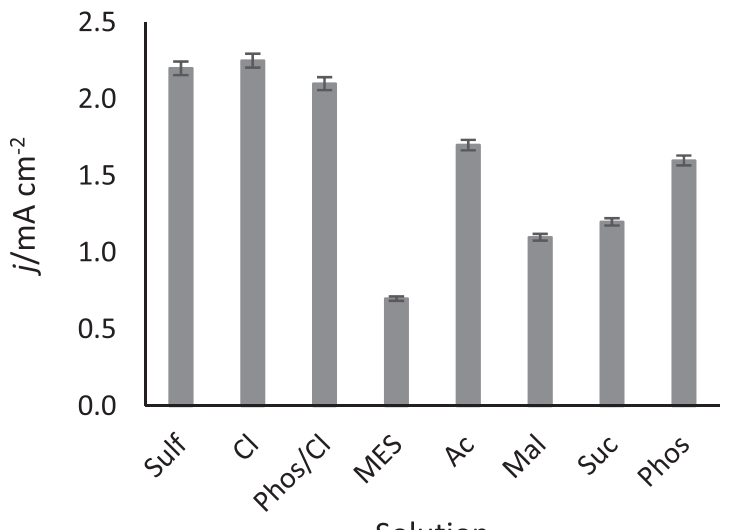

Solution

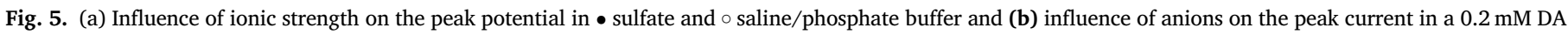
solution with an ionic strength of $0.1 \mathrm{M}$.

peak potentials, there is a significant difference between the peak potentials in the sulfate and phosphate solutions. This is consistent with the buffering action of the phosphate system which prevents significant changes in the interfacial $\mathrm{pH}$ as the dopamine is oxidised. It is well documented that the peak potential is dependent on the $\mathrm{pH}$ and increases with decreasing $\mathrm{pH}$ values [36], consistent with the Nernst equation expression, Eq. (3). In this analysis, DOQ represents the oxidised product, dopamine-o-quinone.

$E=E^{\mathrm{o}}+\frac{0.0591}{n} \log \frac{[D O Q]\left[H^{+}\right]^{2}}{[D A]}$

In the absence of a buffering system, the interfacial $\mathrm{pH}$ will decrease and this will result in local acidification within the diffusion layer at the polymer interface and within the porous polymer matrix. Consequently, higher peak potentials are observed. Indeed, on adding sulfate to the phosphate buffer and maintaining the ionic strength fixed, identical peak potential values were observed. For example, when $0.1 \mathrm{M} \mathrm{Na}_{2} \mathrm{SO}_{4}$ was added to a $0.1 \mathrm{M}$ PBS solution and compared with a solution made using $0.3 \mathrm{M}$ PBS, an identical peak potential of $0.38 \mathrm{~V}$ vs SCE was observed. The influence of the nature of the anions on the peak oxidation current at a fixed ionic strength of $0.1 \mathrm{M}$ and at a pH of 7.0 is illustrated in Fig. 5(b). In this case, the solutions that contain carboxylate groups give rise to lower peak currents, while nearly identical currents are observed with the chloride and sulfate-containing solutions. It is known that the electrochemistry of DA is influenced by the presence of carboxylate anions, and although this is poorly understood, it has been suggested that the carboxylic anions form a product with the DA oxidation species [37]. The MES buffer gives the lowest peak current and this is probably related to the low conductivity of this buffering system. These results clearly illustrate that sulfate, chloride or a saline-based phosphate buffer system is suitable for the detection of DA at the PPy-S $\beta C D$ films.

\subsection{Sensitivity studies}

It is evident from Fig. 2 that the presence of the sulfonated cyclodextrin as a dopant gives rise to a film with a relatively high capacitance. The capacitance of the PPy-S $\beta C D$ film is consistent with studies reported by Suematsu et al. [38], who explained the high capacitance of polypyrrole films doped with sulfonated naphthalene rings to the presence of free sulfonate groups. As the sulfonated $\beta$-cyclodextrin is charged, with 7-11 negatively charge $-\mathrm{SO}_{3}^{-}$groups, then it is indeed possible that some of these sulfonate groups are free, giving rise to the high capacitance of this film. This large charging capacitance of the PPy-S $\beta C D$ film, leads to significant background currents in the cyclic voltammograms, making it difficult to employ voltammetry to determine the sensitivity of the sensor. However, these background currents can be minimised using constant potential amperometry (CPA). In the CPA experiments, a constant potential of $0.65 \mathrm{~V}$ vs SCE was applied and the current was recorded until a steady state current (approximately $30 \mathrm{~min}$ ) was achieved in a $0.1 \mathrm{M} \mathrm{NaCl}$ solution that was agitated by rotating the PPy-S $\beta C D$ modified electrode at $2000 \mathrm{rpm}$. Then $50 \mu \mathrm{L}$ aliquots of a DA stock solution were added. The response time (taken as the time required for the current to increase from $10 \%$ to $80 \%$ of its final value) was less than $2.1 \mathrm{~s}$. A typical calibration curve is shown in Fig. 6 , where the linear region extends to $50 \mu \mathrm{M}$. The regression equation was $I_{\mathrm{p}}=0.90 c_{\mathrm{DA}}$, with a correlation coefficient of 0.999 . This gives a current to concentration ratio, sensitivity, of $0.90 \mu \mathrm{A} \mu \mathrm{M}^{-1} \mathrm{~cm}^{-2}$. Using the linear calibration curve, the limit of detection (LOD) was found to be $1.0 \times 10^{-6} \mathrm{M}$ dopamine. This was obtained using the expression $C_{\mathrm{m}}=3 S_{\mathrm{b}} / \mathrm{m}$, where $C_{\mathrm{m}}$ is the detection limit, $S_{\mathrm{b}}$ is the standard deviation of the blank response and $m$ is the slope of the linear calibration curve. Although this LOD value is not sufficient for a viable in vivo DA sensor, that could follow the concentration variations of DA released from synaptic vesicles in the brain, it is sufficient to detect DA in pharmaceutical products. Indeed, Ferreira et al. [39], used a sensor with a limit of detection of $4.0 \times 10^{-5} \mathrm{M}$ in the analysis of DA in a pharmaceutical mixture.

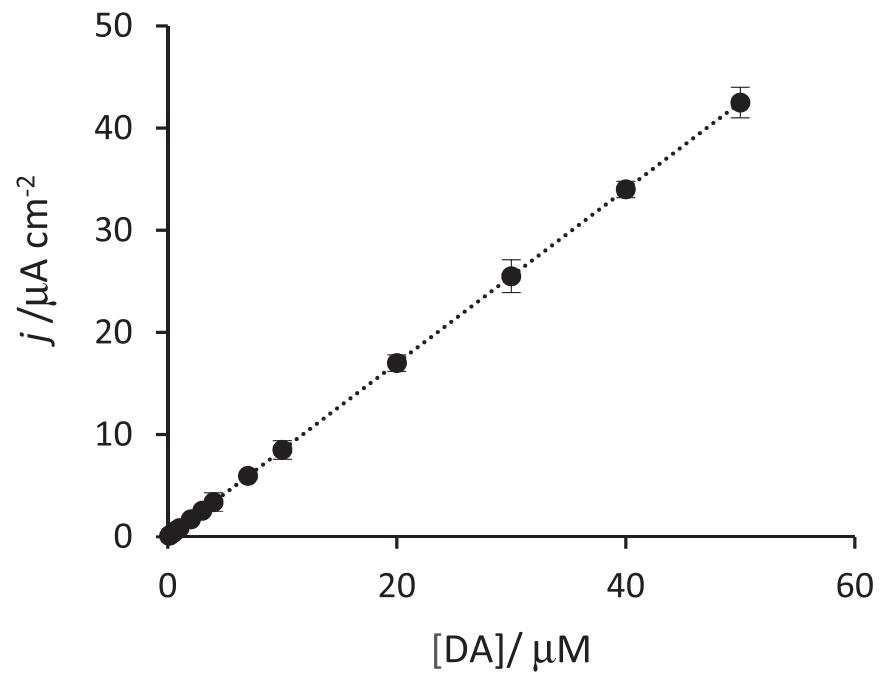

Fig. 6. Calibration curve recorded using constant potential amperometry at $0.65 \mathrm{~V}$ vs SCE in a $0.1 \mathrm{M} \mathrm{NaCl}$ solution. 


\subsection{Selectivity studies}

Studies on the selectivity of the sensor were carried out in the presence of a range of potential interference compounds which were added to DA solutions at fixed concentrations. Cyclic voltammograms were recorded in the mixed DA and interference solution and compared to the corresponding pure DA solution. These data are summarised in Table 1, where the peak current and peak potentials are shown for two DA concentrations in the presence and absence of the interference compounds. The interference compounds included ascorbic acid (AA), uric acid (UA), aspartic acid (Asp), acetylcholine (Ach), aminobutyric acid (GABA), glutamic acid (Glu), glycine (Gly), histamine (HA), acetaminophenol (ACOP) (paracetamol), 5-hydroxytryptamine (5-HT), 5-hydroxyindole acetic acid (5-HIAA), 3,4-dihydroxyphenylacetic acid (DOPAC) and epinephrine (Ep). These compounds were selected as they are all related in some way with the dopaminergic system. Indeed, the analgesic actions of ACOP, more commonly known as paracetamol, are also connected with the dopaminergic system, giving rise to an increase in DA levels in the brain on administration. The values reported in the table are averaged over at least five determinations. It can be clearly negative charge. Furthermore, some of the $-\mathrm{SO}_{3}^{-}$pendants are likely to be free and not involved in charge compensation giving an overall negatively charged interface that attracts dopamine but repels the anionic UA and $\mathrm{AA}$.

Further evidence for the significance of the charge was obtained by selecting molecules that are predominately anionic or cationic. Aspartic acid and glutamic acid are both electroactive and predominately anionic at the $\mathrm{pH}$ of the experiment. Both aspartic acid and glutamic acid are amino acids and depending on the $\mathrm{pH}$ of the solution, they can exist in different forms, as shown in Eq. (4), which in this case represents the aspartic acid. Both amino acids will exist in the zwitterionic form at a pH of 7.0 where the carboxylic groups are ionised to give a polar structure that is predominately anionic. Oxidation of these amino acids was observed at a bare platinum electrode between 0.40 and $0.70 \mathrm{~V}$ vs SCE, while a reduction wave was observed centred at $0.23 \mathrm{~V}$ vs SCE. These potentials are similar to those at which DA is oxidised at the PPy-S $\beta C D$ film, Fig. 2. However, as shown in Table 1 there is no evidence of any interference from the oxidation of these anionic species at the PPy-S $\beta C D$ film. Again, this can be attributed to the negative charge provided by the $-\mathrm{SO}_{3}^{-}$pendants.

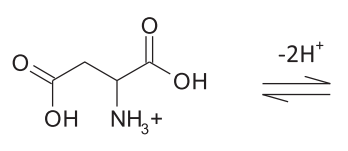

$\mathrm{pH}<2.1$

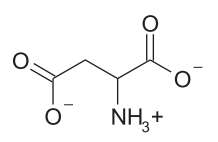

$2.1<\mathrm{pH}<9.8$

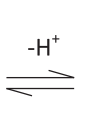

$\overbrace{\mathrm{O}^{-}}^{\mathrm{O}}$

$\mathrm{pH}>9.8$ seen that the DA oxidation peak current and peak potential are not affected by the presence of AA or UA. Regardless of the concentration of DA, there is no evidence of any change in the magnitude of the peak oxidation current. The $\mathrm{pKa}$ value of $\mathrm{UA}$ is 5.4, while the $\mathrm{pKa}$ value of AA is 4.10. At the $\mathrm{pH}$ of the experiment, $\mathrm{AA}$ will exist as an anion, while the UA will again be predominantly in the anionic form. On the other hand, $\mathrm{DA}$ is cationic. As the $\beta$ cyclodextrin has 7 to 11 anionic $-\mathrm{SO}_{3}^{-}$groups as pendants on the rim of the $\mathrm{CD}$ cavity, then it will provide a high local

Table 1

Peak current and peak potentials obtained using cyclic voltammetry recorded at $100 \mathrm{mV} \mathrm{s}^{-1}$ in DA solutions in the absence and presence of interference compounds $(\mathrm{n}=5)$.

\begin{tabular}{|c|c|c|c|c|}
\hline \multirow[t]{2}{*}{ Solution/1.0 mM } & \multicolumn{2}{|c|}{$7.0 \times 10^{-5} \mathrm{M} \mathrm{DA}$} & \multicolumn{2}{|c|}{$2.0 \times 10^{-4} \mathrm{M} \mathrm{DA}$} \\
\hline & $j_{\mathrm{p}} / \mathrm{mA} \mathrm{cm}^{-2}$ & $E_{\mathrm{P}} / \mathrm{mV}$ & $j_{\mathrm{p}} / \mathrm{mA} \mathrm{cm}^{-2}$ & $E_{\mathrm{P}} / \mathrm{mV}$ \\
\hline No Interference & $1.89 \pm 0.05$ & $429 \pm 2$ & $2.21 \pm 0.06$ & $438 \pm 2$ \\
\hline $\mathrm{AA}(1 \mathrm{mM})$ & $1.89 \pm 0.05$ & $429 \pm 3$ & $2.21 \pm 0.06$ & $438 \pm 2$ \\
\hline $\mathrm{AA}(10 \mathrm{mM})$ & $1.89 \pm 0.04$ & $429 \pm 3$ & $2.20 \pm 0.06$ & $437 \pm 2$ \\
\hline UA & $1.89 \pm 0.03$ & $429 \pm 2$ & $2.21 \pm 0.06$ & $438 \pm 3$ \\
\hline Asp & $1.89 \pm 0.05$ & $429 \pm 2$ & $2.21 \pm 0.05$ & $437 \pm 2$ \\
\hline Ach & $1.89 \pm 0.04$ & $429 \pm 3$ & $2.21 \pm 0.06$ & $438 \pm 2$ \\
\hline GABA & $1.89 \pm 0.05$ & $429 \pm 3$ & $2.21 \pm 0.05$ & $438 \pm 2$ \\
\hline Glu & $1.89 \pm 0.04$ & $429 \pm 1$ & $2.21 \pm 0.07$ & $438 \pm 1$ \\
\hline Gly & $1.89 \pm 0.06$ & $429 \pm 1$ & $2.21 \pm 0.05$ & $438 \pm 2$ \\
\hline HA & $1.89 \pm 0.06$ & $428 \pm 3$ & $2.20 \pm 0.07$ & $438 \pm 2$ \\
\hline ACOP & $1.88 \pm 0.03$ & $428 \pm 2$ & $2.21 \pm 0.06$ & $438 \pm 2$ \\
\hline 5-HT & $1.89 \pm 0.04$ & $429 \pm 4$ & $2.21 \pm 0.06$ & $438 \pm 2$ \\
\hline 5-HIAA & $1.89 \pm 0.03$ & $429 \pm 4$ & $2.20 \pm 0.06$ & $438 \pm 4$ \\
\hline Ep & $2.71 \pm 0.07$ & $473 \pm 5$ & $3.14 \pm 0.08$ & $476 \pm 3$ \\
\hline DOPAC & $2.21 \pm 0.06$ & $450 \pm 4$ & $2.25 \pm 0.09$ & $470 \pm 4$ \\
\hline
\end{tabular}

Abbreviations: AA (ascorbic acid), UA (uric acid), Asp (aspartic acid) Ach (acetylcholine), GABA (aminobutyric acid), Glu (glutamic acid), Gly (glycine), HA (histamine), ACOP (paracetamol), 5-HT (5-hydroxytryptamine), 5-HIAA (5-hydroxyindole acetic acid), DOPAC (3,4-dihydroxyphenylacetic acid) and Ep (epinephrine).
Histamine, acetylcholine and 5-hydroxytryptamine are cationic at the $\mathrm{pH}$ of the experiment, and again there is no evidence of any interference from these molecules, as shown in Table 1. Although these molecules may be attracted to the negatively charged cyclodextrin, they are not detected at the PPy-S $\beta C D$ film at the concentrations shown in the table. However, some interference was seen with 5-hydroxytryptamine using higher DA concentrations of $0.5 \mathrm{mM}$ and $1.0 \mathrm{mM}$. When epinephrine (Ep), which will exist as a positively charged species at the $\mathrm{pH}$ of the experiment as the $\mathrm{pKa}$ value is approximately 9.8 , is added to the DA-containing solution interference is observed, Table 1 . These results obtained with the histamine and Ep show that while charge plays a significant role, the structure of the molecule is also of significance. Ep is closely related to the structure of DA, being a catecholamine, while the structure of histamine, although possessing a protonated amine, is very different. The interference observed with Ep is shown more clearly in Fig. 7, where it is seen that the level of interference is more significant at the lower DA concentrations, indicating competition between the DA and Ep molecules. Similarly, DOPAC, which has a structure that is similar to $\mathrm{DA}$, gives rise to significant interference, with a clear increase in the oxidation current and the oxidation peak potential.

\subsection{Mode of detection}

It is clear from the results presented that the $P P y-S \beta C D$ film gives reasonably good sensitivity and very good selectivity and this is connected to the $S \beta C D$ dopant. In order to determine if the oxidation of DA at the $P P y-S \beta C D$ film was under diffusion control or an adsorption process, voltammograms were recorded at different scan rates and the peak currents arising from the oxidation of DA were recorded. It is evident form Fig. 8 that the Randles-Sevcik equation, Eq. (5), is satisfied, giving a linear relationship between the peak current density and the square root of the scan rate. In this analysis, $j_{\mathrm{p}}$ is the peak current density, $n$ is the electron stoichiometry, $D$ is the diffusion coefficient, $v$ is the scan rate and $C_{\mathrm{o}}$ is the concentration. This linear relationship is consistent with a 


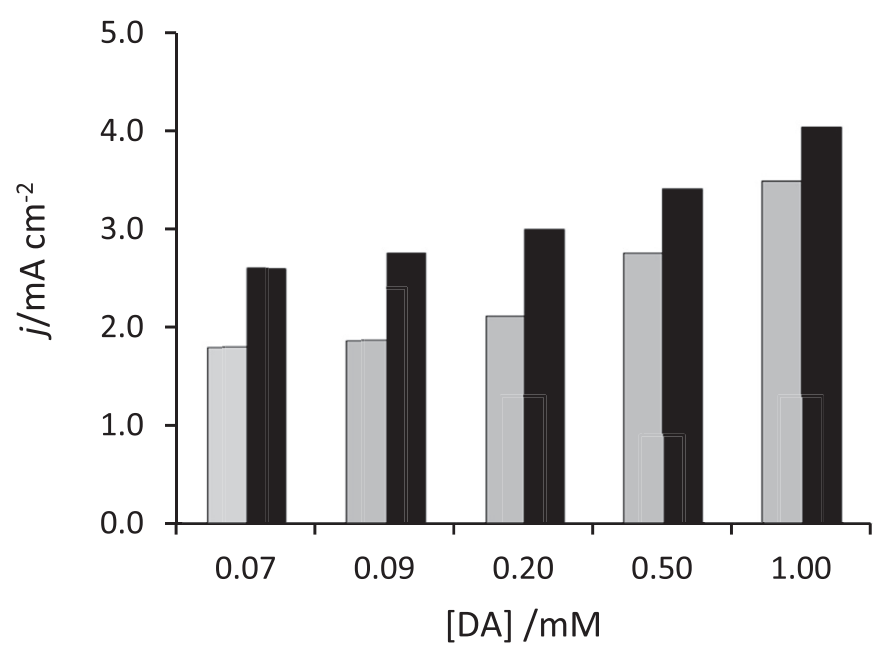

Fig. 7. Peak current density as a function of the DA concentration in the absence (grey) and presence (black) of $1.0 \mathrm{mM}$ Ep.

diffusion-controlled process and the diffusion coefficient of DA was calculated as $3.6 \times 10^{-6} \mathrm{~cm}^{2} \mathrm{~s}^{-1}$. Indeed, on plotting the logarithm of the current as a function of the logarithm of the scan rate, a linear plot with a gradient of 0.5 was obtained, as illustrated in the inset of Fig. 8, indicating a pure diffusion-controlled process.

$j_{p}=\left(2.69 \times 10^{5}\right) n^{3 / 2} D^{1 / 2} v^{1 / 2} C_{\mathrm{o}}$

The potential interactions between DA and the PPy-S $\beta C D$ film were probed by fitting the data to Michaelis-Menten kinetics, which is summarised in Eqs. (6)-(8). The enzyme (E) binds to a substrate (S) to form a complex $(\mathrm{E}-\mathrm{S})$, which then dissociates to generate the free enzyme and the product (P), Eq. (6). In Eq. (7), the rate of substrate conversion, V, is related to the maximum rate of substrate conversion, $V_{\max }$, the substrate concentration, [S], and $K_{\mathrm{m}}$, which gives the Michaelis-Menten constant. A linear transformation of the Michaelis-Menten equation gives the Lineweaver-Burk equation, Eq. (8).

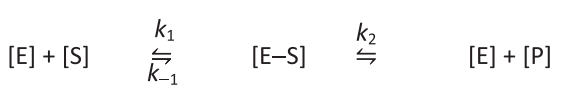

$V=\frac{V_{\max }[S]}{K_{m}+[S]}$

$\frac{1}{V}=\frac{1}{V_{\max }}+\frac{K_{m}}{V_{\max }[S]}$

These equations were employed by setting $V$ to the current density, $j$, and consequently $V_{\max }$ is equal to $j_{\max }$. The DA oxidation current density was obtained from amperometry measurements at a fixed potential of $0.65 \mathrm{~V}$ vs SCE with rotation of the electrode at $2000 \mathrm{rpm}$. It is evident from Fig. 9 that the data obey the Michaelis-Menten and Lineweaver-Burk equations. However, the Michaelis-Menten curve does not approach a true $j_{\max }$ even at DA concentrations of $1.0 \mathrm{mM}$. The Michaelis constant, $K_{\mathrm{m}}$, and the maximum current, $j_{\max }$, were estimated as $1.53 \times 10^{-3} \mathrm{M}$ and $1.17 \times 10^{-3} \mathrm{~A} \mathrm{~cm}^{-2}$, respectively, indicating a weak interaction between DA and the PPy-S $\beta C D$ film. Using the Lineweaver-Burk relationship, the $K_{\mathrm{m}}$ and $j_{\max }$ were estimated as $6.34 \times 10^{-4} \mathrm{M}$ and $5.89 \times 10^{-4} \mathrm{~A} \mathrm{~cm}^{-2}$, respectively. Again, these values indicate a weak binding interaction between PPy-S $\beta C D$ and DA. This is consistent with the formation of a weak inclusion complex between DA and the $S \beta C D$ dopant where the hydrophobic ring of DA is included within the cyclodextrin cavity and the protonated amine group is maintained outside the cavity and electrostatically attracted to the anionic sulfonated groups on the rim of the cyclodextrin cavity [40].

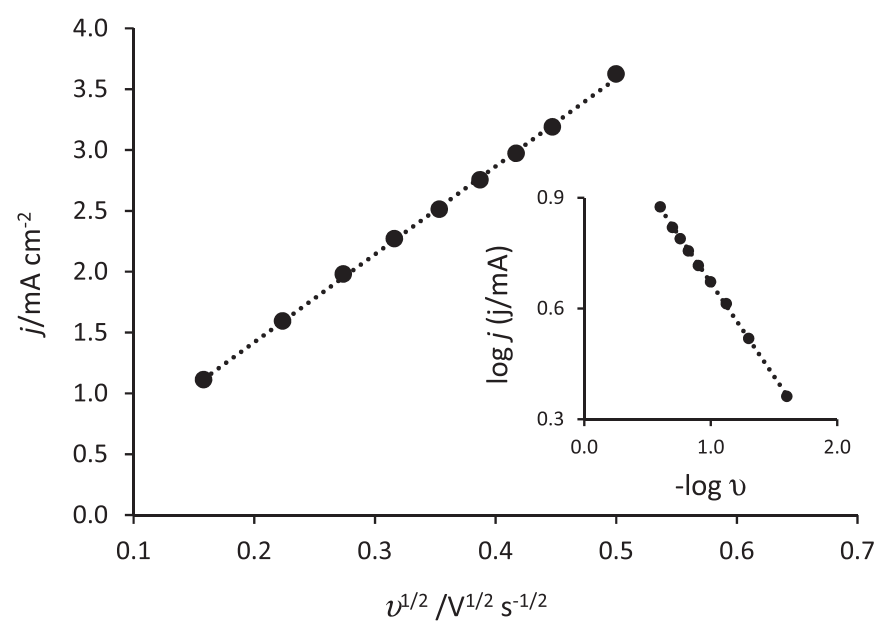

Fig. 8. Peak current (oxidation of DA) plotted as a function of the square root of scan rate and inset shows a plot of the logarithm of peak current as a function of the logarithm of scan rate.

This analysis is consistent with the good selectivity. However, selectivity is not achieved with molecules that have structures that are similar to dopamine, suggesting that these structurally related molecules also bind weakly with the S $\beta C D$. To test this hypothesis, rotating disc voltammetry was used to probe the potential interactions between epinephrine and $S \beta C D$ in solution. Typical voltammograms are shown in Fig. 10, where the rotating disc voltammograms were recorded in a $0.5 \mathrm{mM}$ Ep solution in the absence and presence of added S $\beta C D$. There is a clear reduction in the limiting currents with increasing S $\beta C D$ concentrations. This suggests that Ep forms an inclusion complex with $S \beta C D$ and the concentration of free Ep is reduced. In addition, as the concentration of $S \beta C D$ increases and equilibrium is shifted in favour of the inclusion complex, the now much larger Ep:S $\beta C D$ inclusion complex species diffuse at a slower rate giving a reduction in the apparent diffusion coefficient of Ep. It is also evident that the half-wave potentials increase with increasing concentrations of $S \beta C D$, indicating that the Ep is more difficult to oxidise when included in S $\beta C D$. Using the Levich equation, Eq (9), the apparent diffusion coefficients, $D$, were evaluated and are plotted as a function of the $S \beta C D$ concentrations in the inset in Fig. 10. In this analysis, $J_{L}$ is the limiting current, $n$ corresponds to the number of electrons transferred, $A$ represents the surface area of the electrode, $D$ is the diffusion coefficient of the analyte (representing both the free and included molecule), $\omega$ is the rotation speed, $v$ is the kinematic viscosity and $C$ is the concentration of analyte. The value of the ratio, $D_{\mathrm{c}} / D_{\mathrm{f}}$, is 0.42 , where $D_{\mathrm{c}}$ corresponds to the diffusion coefficient with a large excess of $S \beta C D$ and $D_{\mathrm{f}}$ represents the diffusion coefficient of the free Ep. This is in very good agreement with ratios found in the literature for the complexation of ferrocenes with neutral cyclodextrins [41].

$J_{L}=(0.62) n F A D^{2 / 3} \omega^{1 / 2} v^{-1 / 6} C$

The complex formation constant, $K$, between Ep and the S $\beta C D$ was determined using Eq. (10). Here, $F$ is the Faraday constant, $R$ is the gas constant, $T$ is the temperature, $\left(E_{1 / 2}\right)_{\text {app }}$ and $\left(E_{1 / 2}\right)_{\mathrm{f}}$ are the half-wave potentials in the presence and absence of the $S \beta C D$, respectively. $K$ is the complex formation constant and $D_{\mathrm{c}}$ and $D_{\mathrm{f}}$ are the diffusion coefficients in the presence and absence of the $S \beta C D$, respectively. A complex formation constant of $194.18 \pm 6.10$ was determined using Eq. (10), which indicates the formation of a weak complex. A slightly higher value of $331.28 \pm 5.85$ was obtained with DA [40]. Using a similar approach, there was no change in the apparent diffusion coefficients of AA or UA in the presence of a large excess of $S \beta C D$, lending support to the idea that the selective oxidation of DA at the PPy-S $\beta C D$ is related to weak interactions between DA and the $S \beta C D$ dopant. 

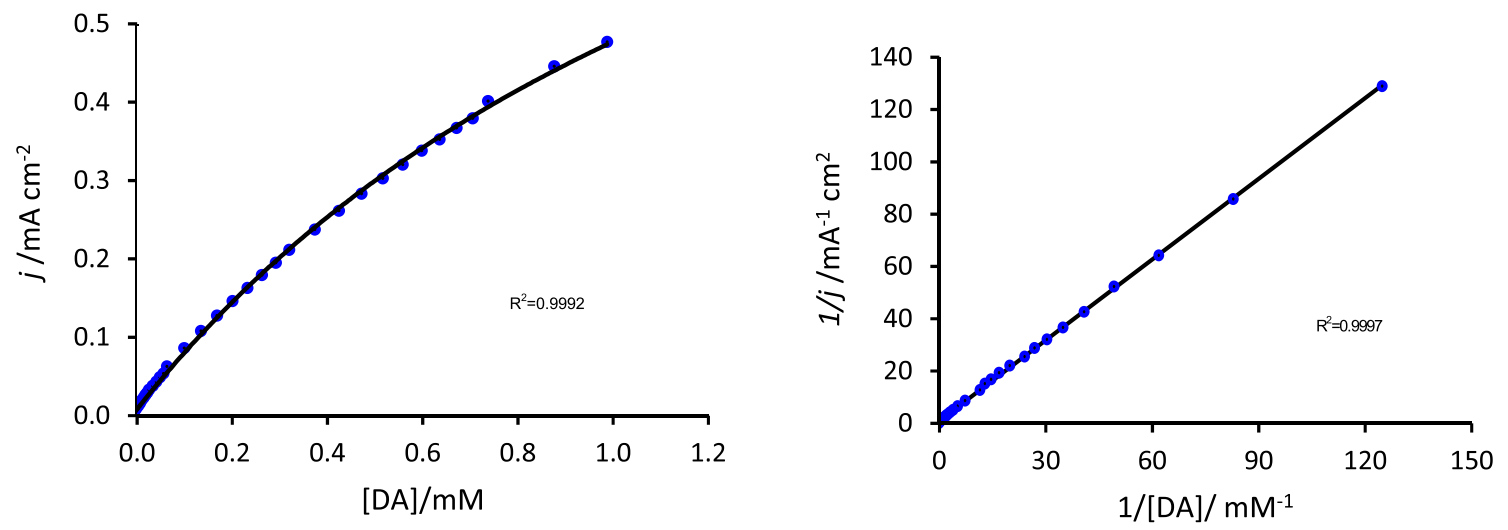

Fig. 9. (a) Michaelis plot of the current recorded at $0.65 \mathrm{~V}$ vs SCE as a function of dopamine concentration and (b) corresponding Lineweaver-Burk plot.

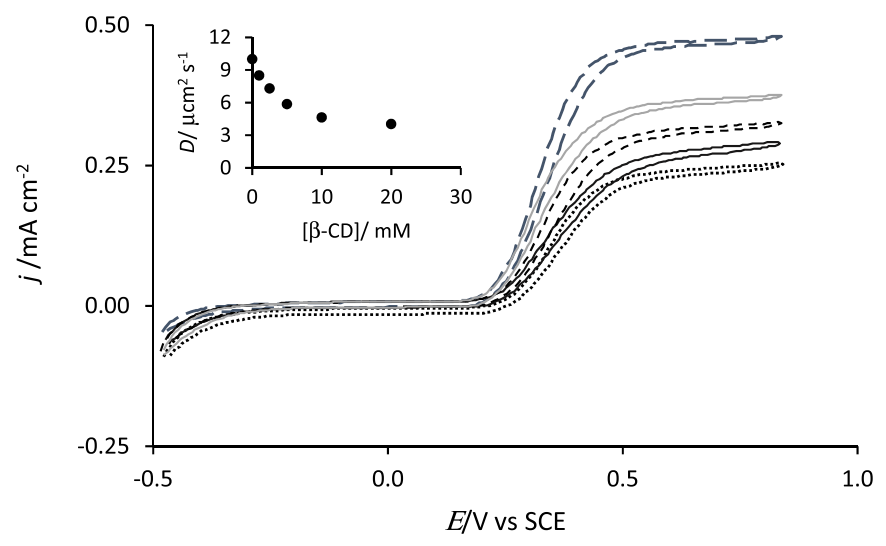

Fig. 10. Rotating disc voltammograms recorded at a GC electrode at $900 \mathrm{rpm}$ in a phosphate buffer solution, $\mathrm{pH}=7.0$ with $0.5 \mathrm{mM}$ Ep and with $-\mathbf{-}-$ no added $S \beta C D=2.5 \mathrm{mM} \mathrm{S} \beta C D,---5 \mathrm{mM} \mathrm{S} \beta C D,-10 \mathrm{mM} \mathrm{S} \beta C D$ and $\bullet \bullet \bullet \bullet-20 \mathrm{mM} \mathrm{S} \beta C D$.

$\frac{F}{R T}\left\{\left(E_{\frac{1}{2}}\right)_{\text {app }}-\left(E_{\frac{1}{2}}\right)_{\mathrm{f}}\right\}=\ln (1+K[S C D])+\ln \left(\frac{D_{c}}{D_{\mathrm{f}}}\right)^{1 / 2}$

\section{Conclusions}

Polypyrrole doped with a sulfonated $\beta$-cyclodextrin was formed at $0.80 \mathrm{~V}$ vs SCE in a $0.2 \mathrm{M}$ pyrrole and $0.01 \mathrm{M}$ cyclodextrin solution. This polymer has good sensitivity in the detection of DA. A linear calibration curve was obtained giving a detection limit of $1.0 \times 10^{-6} \mathrm{M}$ and a sensitivity of $0.90 \mu \mathrm{A} \mu \mathrm{M}^{-1} \mathrm{~cm}^{-2}$. Excellent selectivity for the detection of dopamine was also achieved in the presence of several interference species, such as ascorbic, uric, aspartic and glutamic acids, and the cationic histamine, acetylcholine and 5-hydroxytryptamine. However, interference was observed with the structurally-related epinephrine and DOPAC. These results are consistent with a weak binding interaction between DA and the cyclodextrin dopant, which may indicate that the formation of a weak inclusion complex, favours the selective oxidation of $\mathrm{DA}$ in the presence of several interference compounds.

\section{Acknowledgements}

This work was funded by the Irish Research Council through a postgraduate award to C.C. Harley and Science Foundation Ireland, Grant RFP/EEB006.

\section{References}

[1] M.L.A.V. Heien, A.S. Khan, J.L. Ariansen, J.F. Cheer, P.E.M. Phillips, K.M. Wassum, R.M. Wightman, Real-time measurement of dopamine fluctuations after cocaine in the brain of behaving rats, Proc. Natl. Acad. Sci. U.S.A. 102 (2005) 10023-10028.
[2] Y. Liu, J. Huang, H. Hou, T. You, Simultaneous determination of dopamine, ascorbic acid and uric acid with electrospun carbon nanofibers modified electrode, Electrochem. Commun. 10 (2008) 1431-1434.

[3] S.S. Shankar, B.E.K. Swamy, U. Chandra, J.G. Manjunatha, B.S. Sherigara, Simultaneous determination of dopamine, uric acid and ascorbic acid with CTAB modified carbon paste electrode, Int. J. Electrochem. Sci. 4 (2009) 592-601.

[4] H.F. Cui, Y.-H. Cui, Y.-L. Sun, K. Zhang, W.-D. Zhang, Enhancement of dopamine sensing by layer-by-layer assembly of PVI-dmeOs and Nafion on carbon nanotubes, Nanotechnology 21 (2010) 215601.

[5] H. Jeong, S. Jeon, Determination of dopamine in the presence of ascorbic acid by Nafion and single-walled carbon nanotube film modified on carbon fiber microelectrode, Sensors 8 (2008) 6924-6935.

[6] Y. Yuan, J. Xia, F. Zhang, Z. Wang, Q. Liu, Nafion/polyaniline/zeolitic imidazolate framework-8 nanocomposite sensor for the electrochemical determination of dopamine, J. Electroanal. Chem. 824 (2018) 147-152.

[7] W. Zhang, J. Zheng, J. Shi, Z. Lin, Q. Huang, H. Zhang, C. Wei, J. Chen, S. Hu, A. Hao, Nafion covered core-shell structured $\mathrm{Fe}_{3} \mathrm{O}_{4} @$ graphene nanospheres modified electrode for highly selective detection of dopamine, Anal. Chim. Acta 853 (2015) 285-290.

[8] J. Zheng, X. Zhou, Sodium dodecyl sulfate-modified carbon paste electrodes for selective determination of dopamine in the presence of ascorbic acid, Bioelectrochemistry 70 (2007) 408-415.

[9] M. Asif, A. Aziz, H. Wang, Z. Wang, W. Wang, M. Ajmal, F. Xiao, X. Chen, H. Liu, Superlattice stacking by hybridizing layered double hydroxide nanosheets with layers of reduced graphene oxide for electrochemical simultaneous determination of dopamine, uric acid and ascorbic acid, Microchim. Acta 186 (2019) 1-11.

[10] N. Diab, D.M. Morales, C. Andronescu, M. Masoud, W. Schuhmann, A sensitive and selective graphene/cobalt tetrasulfonated phthalocyanine sensor for detection of dopamine, Sens. Actuators, B 285 (2019) 17-23.

[11] T.M. Prado, A. Carrico, F.H. Cincotto, O. Fatibello-Filho, F.C. Moraes, Bismuth vanadate/graphene quantum dot: a new nanocomposite for photoelectrochemical determination of dopamine, Sens. Actuators, B 285 (2019) 248-253.

[12] N.G. Shang, P. Papakonstantinou, M. McMullan, M. Chu, A. Stamboulis, A. Potenza, S.S. Dhesi, H. Marchetto, Catalyst-free efficient growth, orientation and biosensing properties of multilayer graphene nanoflake films with sharp edge planes, Adv. Funct. Mater. 18 (2008) 3506-3514.

[13] Z. Zhuang, J. Li, R. Xu, D. Xiao, Electrochemical detection of dopamine in the presence of ascorbic acid using overoxidised polypyrrole/graphene modified electrodes, Int. J. Electrochem. Sci. 6 (2011) 2149-2161.

[14] J. Mathiyarasu, S. Senthikumar, K.L.N. Phani, V. Yegnaraman, PEDOT-Au nanocomposite film for electrochemical sensing, Mater. Lett. 62 (2008) 571-573.

[15] T. Qian, C. Yu, X. Zhou, S. Wu, J. Shen, Au nanoparticles decorated polypyrrole/ reduced graphene oxide hybrid sheets for ultrasensitive dopamine detection, Sens. Actuators, B 193 (2014) 759-763.

[16] G. Erdogdu, H.B. Mark, E.A. Karagozler, Voltammetric resolution of ascorbic acid and dopamine at conducting polymer electrodes, Anal. Lett. 29 (1996) 221-231.

[17] A.M. Farrington, J.M. Slater, Prediction and characterization of the charge/size exclusion properties of over-oxidised poly(pyrrole) films, Electroanalysis 9 (1997) $843-847$.

[18] A. Ciszewski, G. Milczarek, Polyeugenol-modified platinum electrode for selective detection of dopamine in the presence of ascorbic acid, Anal. Chem. 71 (1999) 1055-1061.

[19] K. Ghanbari, S. Bonyadi, An electrochemical sensor based on reduced graphene oxide decorated with polypyrrole nanofibers and zinc oxide-copper oxide $\mathrm{p}-\mathrm{n}$ junction heterostructures for the simultaneous voltammetric determination of ascorbic acid, dopamine, paracetamol, and tryptophan, New J. Chem. 42 (2018) 8512-8523.

[20] Z. Rui, W. Huang, Y. Chen, K. Zhang, Y. Cao, J. Tu, Facile synthesis of graphene/ polypyrrole 3D composite for a high-sensitivity non-enzymatic dopamine detection, J. Appl. Polym. Sci. 134 (29) (2017) 44840.

[21] H. Mao, J. Liang, H. Zhang, H.Q. Pei, D. Liu, S. Wu, Y. Zhang, X.-M. Song, Poly(ionic liquids) functionalised polypyrrole/graphene oxide nanosheets for electrochemical sensor to detect dopamine in the presence of ascorbic acid, Biosens. Bioelectron. 70 (2015) 289-298. 
[22] M. Wang, M. Cui, W. Liu, X. Liu, Highly dispersed conductive polypyrrole hydrogels as sensitive sensor for simultaneous determination of ascorbic acid, dopamine and uric acid, J. Electroanal. Chem. 832 (2019) 174-181.

[23] G. Bidan, C. Lopez, F. Mendes-Viegas, E. Vieil, A. Gadelle, Incorporation of sulfonated cyclodextrins into polypyrrole: an approach for the electro-controlled delivering of neutral drugs, Biosens. Bioelectron. 10 (1995) 219-229.

[24] K.R. Temsamani, H.B. Mark, W. Kutner, A.M. Stalcup, A simple one-step electrosynthesis of poly(pyrrole-sulfated $\beta$-cyclodextrin) films, J. Solid State Electrochem. 6 (2002) 391-395.

[25] U. Tamer, C. Kanbeş, N. Ertaş, Branched fibers of conducting polypyrrole: synthesis and characterization, Int. J. Polym. Anal. Charact. 14 (2009) 259-270.

[26] V. Annibaldi, C.B. Breslin, The electrochemistry of viologens at polypyrrole doped with sulfonated cyclodextrins, J. Electroanal. Chem. 832 (2019) 399-407.

[27] G.M. Hendy, C.B. Breslin, The incorporation and controlled release of dopamine from a sulfonated $\beta$-cyclodextrin-doped conducting polymer, J. Polym. Res. 26 (3) (2019) 1-8.

[28] P.M. George, A.W. Lyckman, D.A. LaVan, A. Hegde, Y. Leung, R. Avasare, C. Testa, P.M. Alexander, R. Langer, M. Sur, Fabrication and biocompatibility of polypyrrole implants suitable for neural prosthetics, Biomaterials 26 (2005) 3511-3519.

[29] A.L. Laza-Knoerr, R. Gref, P. Couvreur, Cyclodextrins for drug delivery, J. Drug Target. 18 (2010) 645-656.

[30] C.C. Harley, A.D. Rooney, C.B. Breslin, The selective detection of dopamine at a polypyrrole film doped with sulfonated beta-cyclodextrins, Sens. Actuators, B 150 (2010) 498-504.

[31] G. Sauerbrey, The use of quartz oscillators for weighing thin layers and for microweighing, Zeitschrift fur physik 155 (1959) 206-222.
[32] M.D. Ingram, H. Staesche, K.S. Ryder, Ladder-doped" polypyrrole: a possible electrode material for inclusion in electrochemical supercapacitors? J. Power Sources 129 (2004) 107-112.

[33] E. Moloney, C.B. Breslin, Formation and properties of polypyrrole doped with an immobile antibiotic, J. Solid State Electrochem. 23 (2019) 2031-2042.

[34] V. Annibaldi, G.M. Hendy, C.B. Breslin, Studies on the formation and properties of polypyrrole doped with ionised $\beta$-Cyclodextrins: influence of the anionic pendants, J. Solid State Electrochem. 23 (2019) 615-626.

[35] E. Ryan, C.B. Breslin, Formation of polypyrrole with dexamethasone as a dopant: its cation and anion exchange properties, J. Electroanal. Chem. 824 (2018) 188-194.

[36] T. Luczak, Electrochemical oxidation of dopamine in the presence of secondary amine. An alternative way for quantitative dopamine determination at a gold electrode, Electroanalysis 20 (2008) 1639-1646.

[37] E. Winter, R.M. de Carvalho, L.T. Kubota, S. Rath, Effect of carboxylate compounds on the electrochemical behavior of dopamine at a mercury electrode, J. Brazilian Chem. Soc. 14 (2003) 564-579.

[38] S. Suematsu, Y. Oura, J. Tsujimoto, H. Kanno, K. Naoi, Conducting polymer films of cross-linked structure and their QCM analysis, Electrochim. Acta 45 (2000) 3813-3821.

[39] M. Ferreira, L.R. Dinelli, K. Wohnrath, A.A. Batista, O.N. Oliveira, Langmuir-Blodgett films from polyaniline/ruthenium complexes as modified electrodes for detection of dopamine, Thin Solid Films 446 (2004) 301-306.

[40] G.M. Hendy, C.B. Breslin, An electrochemical study in aqueous solutions on the binding of dopamine to a sulfonated cyclodextrin host, Electrochim. Acta 59 (2012) 290-295.

[41] E. Coutouli-Argyropoulou, A. Kelaidopoulou, C. Sideris, G. Kokkinidis, Electrochemical studies of ferrocene derivatives and their complexation by $\beta$-cyclodextrin, J. Electroanal. Chem. 477 (1999) 130-139. 